\title{
Weiterbildendes Studium »Deutsch als Zweitsprache und Mehrsprachigkeit» an der Universität Paderborn
}

\section{Ausgestaltung und Evaluation einer Professionalisierungsmaßnahme im Bereich Inklusion}

Lisa Berkel-Otto, Lydia Böttger, Nele Fischer, Tetyana Vasylyeva,
Svenja Hammer \& Constanze Niederhaus

Journal für Psychologie, 27(2), 237-262

https://doi.org/10.30820/0942-2285-2019-2-237

www.journal-fuer-psychologie.de

\section{Zusammenfassung}

Eine Facette von Vielfalt im schulischen Kontext und damit einen Aspekt von Inklusion stellt migrationsbedingte Mehrsprachigkeit dar. Die weit verbreitete Diskussion um Deutsch als Zweitsprache und durchgängige Sprachbildung kann daher als Teil der Diskussion um Inklusion betrachtet werden und hat wie diese eine hohe Relevanz für Lehrkräfte.

Dieser Beitrag zeigt auf, über welchen Grad an Zufriedenheit mit dem weiterbildenden Studienprogramm berichtet wird und inwieweit sich die Deutsch-als-ZweitspracheKompetenzen und Überzeugungen der an dieser Weiterbildung teilnehmenden Lehrkräfte entwickelt haben. Neben einer Zufriedenheitsumfrage wurden die Kompetenzen in einem Prä-Post-Design mit einem Paper-Pencil-Test erfasst und die Überzeugungen mit einem entsprechenden Fragebogen erhoben.

Vor dem Hintergrund der Forderung nach innovativen und wirksamen Lehrangeboten liefert der Beitrag inhaltliche und konzeptuelle Einblicke für Ausgestaltungsmöglichkeiten und deren Evaluation und somit Impulse für die (Weiter-)Entwicklung von Lerngelegenheiten in der Lehreraus- und -fortbildung, vor allem im Bereich Deutsch als Zweitsprache und Sprachbildung.

Schlüsselwörter: Lehrkräfteweiterbildung, Mehrsprachigkeit, Inklusion, Zufriedenheit, Kompetenzmessung, Überzeugungen 


\section{Summary}

Further education course »German as a Second Language and Multilingualism « at the University of Paderborn. Design and evaluation of a professionalization measure in the field of inclusion

One of the aspects of diversity in the context of school and education and therefore a facet of inclusion is migration-related multilingualism. That is the reason why the professionalization of well-qualified teachers in the field of teaching multilingual learners is more important than ever.

This article shows if and how satisfied the participating teachers are with the course program. It also indicates how far the competencies and beliefs regarding multilingual learners in the professional development program $\gg$ German as a foreign and second language and multilingualism « develop throughout the program. A survey on satisfaction has been conducted. Data concerning competencies have been collected with a paper and pencil test, beliefs with a questionnaire.

The article provides content-related and conceptual insights for different learning settings and their evaluation and therefore serves the demand for innovative and effective course concepts. Hence, it offers new ideas and impulses for (further) development of learning opportunities for professional development and also teacher training.

Key words: teacher training, multilingualism, inclusion, satisfaction, competence measurement, beliefs

\section{$1 \quad$ Einleitung}

Die UN-Konvention über die Rechte von Menschen mit Behinderung1, die seit dem Jahr 2009 rechtskräftig ist, zielt(e) unter anderem auf die Umsetzung eines inklusiven Schulsystems ab. Wird von einem breiten Inklusionsbegriff ausgegangen, der Diversitäten auf allen Ebenen als Normalfall konstituiert, so sind migrationsbedingte Mehrsprachigkeit und sprachlich heterogene Klassenzimmer als Teil eines inklusiven Konzeptes anzusehen (vgl. hierzu auch Riemer 2017). Um die Lehrkräfte für die sich daraus ergebenden hohen Anforderungen zu qualifizieren, wurden in den vergangenen Jahren in allen drei Phasen der Lehrkräftebildung (Ausbildung bzw. Studium, Referendariat und Fort- und Weiterbildung) etliche Angebote geschaffen (siehe für einen ausführlichen Überblick Baumann 2017; Lütke 2017). Im Bundesland NordrheinWestfalen (NRW) ${ }^{2}$ etablierte sich beispielsweise das Modul »Deutsch für Schülerinnen und Schüler mit Zuwanderungsgeschichte (DSSZ) «, welches alle Lehramtsstudierenden durchlaufen. In der dritten Phase der Lehrerbildung wurden ebenfalls Angebote konzipiert. So richtete das Ministerium für Kultur und Wissenschaft (MKW) des Lan- 
des NRW an allen lehrerbildenden Hochschulen Weiterbildungsstudiengänge (WBS) im Bereich »Mehrsprachigkeit, Deutsch als Zweit- und Fremdsprache « ein.

In diesem Zuge wurde auch an der Universität Paderborn ein WBS für Lehrkräfte, die an allgemein- und berufsbildenden Schulen unterrichten, eingerichtet, das sich über die Dauer eines Semesters erstreckt und einen Arbeitsaufwand von 180 Stunden (sechs Credit Points ${ }^{3}$ ) umfasst. Neben Präsenzsitzungen und Workshops zu verschiedenen Themen wie Bedeutung von migrationsbedingter Mehrsprachigkeit, methodisch-didaktische Prinzipien des DaF/DaZ- und des sprachbildenden Unterrichts, Grundlagen des Zweitspracherwerbs und der Alphabetisierung, Sprachstandsdiagnostik, Traumapädagogik und Lehrmaterialien absolvieren die Teilnehmenden auch daran anknüpfende Online-Aufgaben, die den direkten Transfer dieser Inhalte auf den eigenen Unterricht ermöglichen.

Um Aussagen über das Konzept dieses WBS an der Universität Paderborn treffen und daraus Weiterentwicklungsmöglichkeiten ableiten zu können, wurden die im Sommersemester 2017 eingeschriebenen Lehrkräfte evaluativ eng begleitet. In einem PräPost-Design wurden die Kompetenzen der Lehrkräfte hinsichtlich des Unterrichtens mehrsprachiger Schülerinnen und Schüler $(\mathrm{SuS})$ mit dem im Projekt »Professionelle Kompetenzen angehender Lehrkräfte (Sek I) im Bereich Deutsch als Zweitsprache (DaZKom) « (Ehmke et al. 2018) entwickelten Testinstrument gemessen. Die Überzeugungen zu Mehrsprachigkeit in der Schule wurden mit einem Fragebogen erhoben (Fischer und Ehmke 2019). Weiterhin wurde eine Umfrage zur Zufriedenheit hinsichtlich verschiedener Faktoren wie Inhalte, Konzeption, Praktikabilität und empfundener Relevanz von Inhalten des WBS durchgeführt.

\section{Theoretischer Hintergrund}

\subsection{Inklusion, Sprache und migrationsbedingte Mehrsprachigkeit}

Bereits vor mehr als zehn Jahren wurde die UN-Konvention über die Rechte von Menschen mit Behinderung unter dem Oberbegriff Inklusion verabschiedet. Dennoch ist bis heute unklar, worauf sich Inklusion ganz genau bezieht.

Eine Ursache hierfür ist die Ko-Existenz eines engen und eines weiten Inklusionsbegriffs. Während ein enger Inklusionsbegriff vor allem Menschen mit speziellen Beeinträchtigungen (UN-Konvention, Artikel 24) fokussiert, umfasst ein weiter Inklusionsbegriff »sämtliche Heterogenitätsdimensionen « (Riemer 2017) und zielt damit

» auf den Abbau von Bildungsbarrieren und volle soziale Partizipation aller Menschen unabhängig von ihrer Herkunft und ihren sozialen, ökonomischen Benachteiligungen, ihrer 
Herkunfts- oder Familiensprache, ihrer Hautfarbe, ihrem Geschlecht, ihren sexuellen und anderen Orientierungen, ihrem Glaubensbekenntnis oder ihrer Behinderung « (ebd., 175),

ab. Der vorliegende Beitrag schließt sich ausdrücklich diesem weiten Inklusionsverständnis an und versteht migrationsbedingte Mehrsprachigkeit bzw. Sprachliche Diversität entsprechend als Heterogenitätsdimension im Kontext von Inklusion. An dieser Stelle sei neben der Dimension Sprachliche Diversität auf die weiteren fünf Dimensionen der Vielfalt Soziale Ungleichheit und Armut, Geschlecht und Gender, Migration, Forced Migration und Flucht, Behinderung und (Hoch-)Begabung (vgl. Biewer, Proyer und Kremsner 2019) verwiesen: Auch wenn der Beitrag insbesondere Aspekte aus der Heterogenitätsdimension der Sprachlichen Diversität fokussiert, sind diese eng verknüpft mit den Dimensionen der sozialen Ungleichheit und Armut, als mögliches Resultat sprachlicher Diversität, und Migration und Flucht.

Abgelehnt werden hingegen Tendenzen, migrationsbedingte Mehrsprachigkeit bzw. sprachliche Diversität »in die Nähe eines Sondertatbestands sonderpädagogischer Förderung zu rücken « (Riemer 2017, 175) oder die Tatsache, Deutsch als Nicht-Erstsprache zu sprechen, als (spezielle) Beeinträchtigung zu betrachten.

Einen Hinweis auf die hohe Relevanz der Professionalisierung von Lehrkräften im Themenfeld migrationsbedingte Mehrsprachigkeit, Deutsch als Zweitsprache und durchgängige Sprachbildung im Kontext von Inklusion liefern neben dem Bestreben nach Inklusion auch Daten der Bildungsforschung: Etliche Studien, darunter auch große Schulleistungsvergleichsstudien wie PISA, haben gezeigt, dass Ungleichheiten bezüglich des sozioökonomischen Hintergrunds oder der Herkunft noch immer nicht von Schule aufgefangen werden können (Klieme et al. 2010; Walter und Stanat 2008; Weis et al. 2018). Damit ist neben dem sozioökonomischen Hintergrund die Herkunft noch immer ein starker Prädiktor für schulischen (Miss-)Erfolg. Weiterhin zeigt sich, dass verzerrte Lehrkräfteerwartungen die Kompetenzentwicklung von Kindern beeinflussen (Sachverständigenrat deutscher Stiftungen 2017) und (angehende) Lehrkräfte im Hinblick auf Differenzsensibilität und Diskriminierungskritik in der Migrationsgesellschaft nur unzureichend qualifiziert sind (Stiftung Mercator 2017).

Das bedeutet für die Lehrkräfteaus- und -weiterbildung im Bereich Deutsch als Zweitsprache, dass als Voraussetzung für einen gelingenden sprachbildenden (Fach)Unterricht Mehrsprachigkeit als Chance und nicht als Hindernis von Lehrkräften wahrund angenommen werden sollte (Gogolin 2008; Tracy 2014). Lehrkräfte müssen für die sprachliche und kulturelle Heterogenität ihrer Lernenden sensibilisiert werden und diese als Normalfall akzeptieren. Nur dann können eine sprachsensible Schulentwicklung (Krumm 2017) sowie die Berücksichtigung sprachlicher Heterogenität, sprachliche Bildung und Förderung als Teil der inklusiven Schule gelingen (Becker-Mrotzek und Roth 2017). 
Bezüglich einer inklusiven Schule bzw. inklusiven Lehrens und Lernens beziehen wir uns im vorliegenden Beitrag auf das Didaktische Modell für inklusives Lehren und Lernen - DiMiLL (Rödel, Frohn und Moser 2019). Hier lässt sich das Thema Sprachbildung und Deutsch als Zweitsprache zum einen in die Ebene gesamtgesellschaftliche Bedingungen (ebd.) einordnen, da ein Zusammenhang von Sprache und gesellschaftlicher Teilhabe besteht. Zum anderen wird es mit Blick auf die Umsetzung sprachbildenden Fachunterrichts auch der Ebene fachdidaktische Bedingungen (Rödel und Lütke 2019, 84 in ebd.) sowie im Hinblick auf Ressourcen und Kooperation der Beteiligten den schulorganisatorischen Bedingungen zugeordnet.

\subsection{Wirkung von Lehrkräfteweiterbildung}

Die Lehrkräftebildung in Deutschland ist in drei Phasen gegliedert. In der ersten Phase besuchen die Studierenden eine Hochschule und absolvieren dort ein theorieorientiertes Lehramtsstudium. Die zweite Phase ist der Vorbereitungsdienst (Referendariat). In NRW dauert diese Phase 18 Monate und ist durch einen hohen Praxisanteil geprägt. Die Lehrkräftefort- und -weiterbildung stellt die dritte Phase dar. Hier bilden sich die Lehrkräfte zu Themen weiter, die ihre Schule oder die eigenen Interessen vorgeben.

Die Erforschung der Wirksamkeit dieser drei Phasen erfolgte bislang unterschiedlich stark. Während zahlreiche Befunde über die erste und zweite Phase vorliegen (Paetsch et al. 2020), rückte die dritte Phase erst in den letzten Jahren in den Fokus der Wirksamkeitsforschung (Lipowsky 2010). Bezüglich der Erforschung der Wirkungsweise von Fortbildungen unterscheidet das Modell von Frank Lipowsky vier Ebenen, die die Reichweite weiterbildender Maßnahmen einordnen.

$>$ Ebene 1 - Die Reaktionen und Einschätzungen der teilnehmenden Lehrpersonen

$>$ Ebene 2 - Erweiterung der Lehrerkognitionen

$>$ Ebene 3-Unterrichtspraktisches Handeln

$>$ Ebene 4-Effekte auf Schüler/innen

Die hier beschriebene Evaluationsstudie liefert einen Beitrag für die Erforschung der Wirksamkeit von Lehrkräfteweiterbildung. Dabei wird zum einen die Ebene 1 fokussiert, indem die Ergebnisse des Einsatzes eines Zufriedenheitsfragebogens dargestellt werden. Zum anderen werden Daten zur Wirkung des Weiterbildungsstudiums auf die Lehrerkognitionen präsentiert, die mittels des DaZKom-Tests und eines BeliefsFragebogens erhoben wurden und die sich auf die Ebene 2 des Modells beziehen. Im Folgenden werden die drei erfassten Elemente Zufriedenheit, Wissen sowie Beliefs näher erläutert. 


\subsubsection{Reaktionen und Einschätzungen der teilnehmenden Lehrpersonen: Zufriedenheit}

Reaktionen und Einschätzungen der teilnehmenden Lehrpersonen auf Fortbildungsmaßnahmen werden häufig erhoben. Das mit Blick auf Lehrerfortbildung entwickelte Modell von Lipowsky (2010) berücksichtigt folgende vier Faktoren, die die Zufriedenheit mit einer Fortbildungsmaßnahme beeinflussen: Der Bezug auf den Unterricht und das Curriculum, der kollegiale Austausch und Partizipationsmöglichkeiten, eine angenehme Atmosphäre sowie die Professionalität der Moderatoren. Aktuelle Studien zeigen allerdings keinen signifikanten Zusammenhang zwischen der Zufriedenheit und der Änderung von Kognitionen sowie einem veränderten beruflichen Handeln der Lehrkräfte (Goldschmidt und Phelps 2007, vgl. den Überblick in Lipowsky und Rzejak 2012, 3). Somit ist die Zufriedenheit der Lehrkräfte kein Indikator für den Fortbildungserfolg. Dennoch ist die Zufriedenheit nicht zu vernachlässigen, da zum einen »ein Minimum an Akzeptanz eine notwendige, wenngleich eben keine hinreichende Voraussetzung für einen erfolgreichen Lern- und Transferprozess darstellt « und sich die Akzeptanz einer Fortbildung zum anderen »eher indirekt - über das Engagement und die Intensität der Nutzung der Fortbildungsangebote durch die Lehrer/innen - auf den Lern- und Transferprozess auswirk(t)«(Lipowsky 2010, 54). Laut Lipowsky und Rzejak (2012) setzt der Erfolg einer Fortbildung häufig längere und meist anstrengende Lernprozesse voraus, sodass Fortbildungen unter Umständen auch Unzufriedenheit, Zweifel oder Irritationen bei Lehrkräften auslösen können, die auch durch das Infragestellen bestehender Überzeugungen durch die Fortbildungsinhalte bedingt sein können.

In der Trainingsforschung hingegen konnten engere Zusammenhänge zwischen der subjektiv empfundenen Relevanz und Nützlichkeit und dem Wissenszuwachs sowie dem beruflichen Handeln aufgezeigt werden (Hagemann und Kluge 2014). Obwohl diese Erkenntnisse nicht in Bezug auf Lehrkräftefortbildungen gewonnen wurden, geht Lipowsky (2014) davon aus, dass sich die empfundene Relevanz auch auf die Motivation der Lehrkräfte auswirkt und möglicherweise sogar eine Schlüsselvariable für deren Wirksamkeit darstellt.

Die vorliegende Untersuchung erfasst daher sowohl die Zufriedenheit als auch die empfundene inhaltliche Relevanz des Weiterbildungsangebotes.

\subsubsection{Erweiterung der Lehrerkognitionen: Wissen}

Der Lipowskys Modell zugrundeliegende Kompetenzbegriff (Lipowsky 2006) geht auf Weinert (2001) zurück und setzt sich aus kognitiven, motivationalen, volitionalen und sozialen Anteilen einer Person zusammen. Die kognitiven Fähigkeiten werden an dieser Stelle noch einmal in Bezug auf Bromme (1997, professionelles Lehrerwissen) um 
Weiterbildendes Studium »Deutsch als Zweitsprache und Mehrsprachigkeit« ...

\begin{tabular}{|c|c|c|c|c|c|}
\hline & \multirow{2}{*}{ Dimension } & \multirow{2}{*}{ Subdimension } & \multicolumn{3}{|c|}{ Facetten } \\
\hline & & & Stufe I & Stufe II & Stufe III \\
\hline \multirow{19}{*}{ 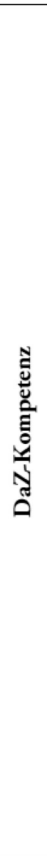 } & \multirow{7}{*}{$\begin{array}{l}\text { Fachregister } \\
\text { (Fokus auf } \\
\text { Sprache) }\end{array}$} & \multirow{4}{*}{$\begin{array}{l}\text { Grammatische } \\
\text { Strukturen und } \\
\text { Wortschatz }\end{array}$} & \multicolumn{3}{|c|}{ Morphologie } \\
\hline & & & \multicolumn{3}{|c|}{ (Lexikalische) Semantik } \\
\hline & & & \multicolumn{3}{|c|}{ Syntax } \\
\hline & & & \multicolumn{3}{|c|}{ Textlinguistik } \\
\hline & & \multirow{3}{*}{$\begin{array}{l}\text { Semiotische } \\
\text { Systeme }\end{array}$} & \multicolumn{3}{|c|}{ Darstellungsformen } \\
\hline & & & \multicolumn{3}{|c|}{$\begin{array}{c}\text { Sprachliche Bezüge zwischen } \\
\text { Darstellungsformen }\end{array}$} \\
\hline & & & \multicolumn{3}{|c|}{ Mündlichkeit vs. Schriftlichkeit } \\
\hline & \multirow{6}{*}{$\begin{array}{c}\text { Mehrsprachig- } \\
\text { keit } \\
\text { (Fokus auf } \\
\text { Lernprozess) }\end{array}$} & \multirow{4}{*}{$\begin{array}{l}\text { Zweitsprach- } \\
\text { erwerb }\end{array}$} & \multicolumn{3}{|c|}{ Interlanguage-Hypothese } \\
\hline & & & \multicolumn{3}{|c|}{$\begin{array}{c}\text { Meilensteine zweitsprachlicher } \\
\text { Entwicklung }\end{array}$} \\
\hline & & & \multicolumn{3}{|c|}{$\begin{array}{c}\text { Gesteuerter vs. Ungesteuerter } \\
\text { Zweitspracherwerb }\end{array}$} \\
\hline & & & \multicolumn{3}{|c|}{ Literacy/Bildungssprache } \\
\hline & & \multirow{2}{*}{ Migration } & \multicolumn{3}{|c|}{ Sprachliche Vielfalt in der Schule } \\
\hline & & & \multicolumn{3}{|c|}{ Umgang mit Heterogenität } \\
\hline & \multirow{6}{*}{$\begin{array}{c}\text { Didaktik } \\
\text { (Fokus auf } \\
\text { Lehrprozess) }\end{array}$} & \multirow{3}{*}{ Diagnose } & \multicolumn{3}{|c|}{ Mikro-Scaffolding } \\
\hline & & & \multicolumn{3}{|c|}{ Makro-Scaffolding } \\
\hline & & & \multicolumn{3}{|c|}{ Umgang mit Fehlern } \\
\hline & & \multirow{3}{*}{ Förderung } & \multicolumn{3}{|c|}{ Mikro-Scaffolding } \\
\hline & & & \multicolumn{3}{|c|}{ Makro-Scaffolding } \\
\hline & & & \multicolumn{3}{|c|}{ Umgang mit Fehlern } \\
\hline
\end{tabular}

Abbildung 1: Kompetenzmodell DaZKom

epistemologische Überzeugungen und selbstbezogene Kognitionen von Lehrpersonen erweitert.

Für die Erfassung von Lehrerkognitionen bzw. Wissen im Bereich Deutsch als Zweitsprache existieren im Bereich der empirischen Kompetenzmessung bislang lediglich zwei Testinstrumente. Während das Projekt SprachKoPF Kompetenzen für den Bereich der Sprachförderung von pädagogischem Fachpersonal im Vorschulbereich fokussiert (Tracy et al. 2014), beschäftigt sich das Projekt DaZKom mit Deutsch als Zweitsprache-Kompetenzen angehender Lehrkräfte (Ehmke et al. 2018).

Im Projekt DaZKom wurde auf Basis einer Analyse von 60 Curricula deutscher Universitäten und Institutionen ein Kompetenzmodell entwickelt, welches Deutsch als Zweitsprache-Kompetenzen angehender Lehrkräfte modelliert (Carlson und Präg 2018; siehe Abbildung 1). Es besteht aus drei Dimensionen, welche sich in Subdimensionen und diese wiederum in unterschiedliche Facetten aufgliedern.

Basierend auf diesem Modell wurden ein Testinstrument entwickelt und Validierungsstudien durchgeführt (Hammer et al. 2015, Hammer und Ehmke 2018), mit dem 
die DaZ-Kompetenzentwicklung angehender und praktizierender Lehrkräfte ermittelt werden kann (Paetsch et al. 2019; Hammer und Berkel-Otto 2019).

Daran anknüpfend wurde ein Standardsetting durchgeführt basierend auf einer Stichprobe von 1.383 Studierenden verschiedener deutscher Universitäten. Ein Expertenrating mit sechs Experten aus dem Bereich Deutsch als Zweitsprache aus der Forschung sowie der Praxis ermittelten hierbei drei Kompetenzstufen, die als Standard gelten sollen (Gültekin-Karakoç 2018):

$>$ Unter Mindeststandard - unspezifischer Ansatz zu DaZ: realisieren beispielsweise unspezifisch die Rolle der Sprache beim Lernen, verfügen über linguistisches Basiswissen und können zwischen konzeptionell mündlicher und schriftlicher Sprache unterscheiden.

$>$ Mindeststandard - für DaZ sensibilisiert: kennen beispielsweise den Zusammenhang zwischen sprachlichem und fachlichem Lernen, haben selektive DaZKenntnisse und erste Ideen zur DaZ-Förderung.

> Regelstandard - über DaZ informiert: kennen beispielsweise Sprachförderelemente und können Unterrichtsinteraktionen, Produktionen von Schülern sowie Lehr- und Lernmaterial analysieren.

\subsubsection{Erweiterung der Lehrerkognitionen: Überzeugungen/Beliefs}

Neben dem Wissen zählen auch beliefs bzw. Überzeugungen zu den Lehrerkognitionen. Der vorliegende Beitrag bezieht sich neben der Bezeichnung beliefs vor allem auf das deutschsprachige Synonym der Überzeugungen (Reusser et al. 2011, 479). Überzeugungen sind angelehnt an das Kompetenzmodell von Baumert und Kunter (2006) ein bedeutender Bestandteil professioneller Kompetenz von Lehrkräften. Sie werden mit verallgemeinernden Konstrukten verglichen, die Handlungsentscheidungen erleichtern sollen (Sigel 1985), und stellen daher für Lehrkräfte, die in ihrem Berufsalltag häufig unter Zeitdruck Entscheidungen treffen bzw. entsprechende Handlungen ergreifen müssen, ein grundlegendes Orientierungsgerüst dar (Blömeke und Oser 2012). Immer mehr Studienergebnisse sprechen dafür, dass der gezielte Einfluss von Wissen zu einer Veränderung von Überzeugungen führen kann (u. a. Hachfeld et al. 2012; Hartwig et al. 2017). Wissen und Überzeugungen werden daher, angelehnt an Blömeke et al. (2008), als sich wechselseitig beeinflussend angesehen.

Die Forschung zu beliefs im Allgemeinen nimmt im deutschsprachigen Raum stetig zu, doch vor allem quantitative Studien zu den Überzeugungen von angehenden und bereits ausgebildeten Lehrkräften hinsichtlich sprachlich-kultureller Heterogenität sind nur vereinzelt zu finden (Riebling 2013; Maak et al. 2015; Hammer et al. 2016; Fischer et al. 2018; Fischer und Ehmke 2019). Ein Großteil der Studien hat zum Ergebnis, dass die Überzeugungen der befragten Lehrkräfte grundsätzlich eine positive 
Tendenz aufzeigen, es aber vor allem in Hinblick auf die praktische Umsetzung von sprachsensiblem Fachunterricht nach wie vor Vorbehalte und Unsicherheiten gibt. Verschiedene Studien fordern daher eine stärkere Reflexion der eigenen Überzeugungen in der Lehrkräfteaus- und -fortbildung (u. a. Wischmeier 2012; Hallitzky und Schliessleder 2008; Fischer 2018).

\section{Forschungsdesign}

\subsection{Forschungsfragen}

Folgende Fragen standen im Fokus dieser Studie:

1. Wie zufrieden sind die Lehrkräfte mit dem WBS? Als wie relevant für die berufliche Praxis werden die Inhalte empfunden?

Vor dem Hintergrund der freiwilligen Teilnahme der Lehrkräfte am WBS bis zu dessen Abschluss wird angenommen, dass sie die Inhalte des WBS für relevant halten und aus diesem Grund mit dem Studium zufrieden sind.

2. Kann für die teilnehmenden Lehrkräfte des WBS ein Wissenszuwachs im Bereich Deutsch als Zweitsprache festgestellt werden?

Vor dem Hintergrund, dass im WBS Inhalte und Konzepte vermittelt werden, die Bestandteil des DaZ-Kompetenzmodells sind, wird von einem Wissenszuwachs ausgegangen.

3. Kann für die teilnehmenden Lehrkräfte des WBS eine positive Veränderung der Überzeugungen zu sprachlich-kultureller Heterogenität in Schule und Unterricht festgestellt werden?

Vor dem Hintergrund, dass Überzeugungen als veränderbar gelten, wird erwartet, dass sich die Überzeugungen der Teilnehmenden durch das WBS positiv entwickeln, da Wissen zu sprachlich-kultureller Heterogenität in Schule und Unterricht vermittelt wurde.

\subsection{Instrumente}

\section{Zufriedenheit}

Aussagen dazu, wie zufrieden die Lehrkräfte mit dem WBS sind und für wie relevant sie die Inhalte halten, wurden mit einem Fragebogen am Ende der Fortbildung erfasst. Der Fragebogen wurde mit dem Ziel, einen möglichen Entwicklungsbedarf für das WBS aufzuzeigen, von den WBS-Dozentinnen zur Veranstaltungsevaluation erarbeitet. Der Fragebogen umfasst sechs Rubriken - WBS allgemein, Begleitung und 
Betreuung durch Dozenten, Seminare mit dem Schwerpunkt Inhalte, Blended-LearningFormat, von externen Dozenten gehaltene Workshops sowie Unterstützung durch das berufliche Umfeld - und enthält insgesamt 26 geschlossene und offene Fragen sowie ein Freitextfeld.

Zufriedenheit wurde anhand von insgesamt sieben Fragen, sechs geschlossenen und einer offenen, in den Rubriken WBS allgemein und Seminare erfasst. Die geschlossenen Fragen adressieren den Aufbau, das inhaltliche Niveau des Studiums, die Atmosphäre in den Seminaren sowie die Erfüllung von Erwartungen an das Studium. Die Teilnehmenden konnten die entsprechenden Fragen anhand von Likert-Skalen mit vier Abstufungen (sehr gut ... schlecht) beantworten. Die Skala zum inhaltlichen Niveau hatte fünf Abstufungen (zu hoch ... zu niedrig). Die offene Frage erfasst Gründe, warum die Erwartungen an das Studium erfüllt bzw. nicht erfüllt wurden. Die inhaltliche Relevanz wurde mithilfe von drei Fragen erfasst. Eine offene Frage verlangt eine Aufzählung von Inhalten, die für die Teilnehmenden relevant waren. Zwei geschlossene Fragen beziehen sich auf die Relevanz der beiden extern angebotenen Workshops und erfragen diese mithilfe vierstufiger Likert-Skalen (sehr relevant ... nicht relevant). Die Teilnehmenden konnten außerdem angeben, dass sie am Workshop nicht teilgenommen haben.

\section{Wissen}

Das Wissen im Bereich Deutsch als Zweitsprache und zum Unterrichten migrationsbedingt mehrsprachiger Schülerinnen und Schüler wurde in der ersten und in der letzten Sitzung der Weiterbildung mithilfe des im Projekt DaZKom entwickelten Paper-PencilTests erhoben. Der Test dauert 40 Minuten und beinhaltet 47 Items (zwölf offen, elf halboffen, 24 geschlossen). Entsprechend dem DaZKom-Modell, welches die drei Dimensionen $\gg$ Fachregister «, » Mehrsprachigkeit « und »Didaktik « umfasst, verteilen sich die Items auf ebendiese.

Insgesamt weist der Test eine EAP/PV-Reliabilität von $\alpha=0,78$ auf. Jedes Item enthält authentisches Stimulusmaterial (Schüler-Lehrer-Interaktionen, authentische Schülertexte, Textaufgaben aus Mathematiklehrwerken, Fallbeispiele). Abbildung 2 zeigt ein Beispielitem.

\section{Beliefs}

Die Überzeugungen zu sprachlich-kultureller Heterogenität in Schule und Unterricht wurden anhand eines empirisch erprobten Instruments erfasst (Fischer und Ehmke 2019). Das Fragebogeninstrument basiert auf einem theoretischen Modell, das aus einer umfassenden Literaturrecherche abgeleitet wurde (Fischer 2018) ${ }^{4}$. Es umfasst insgesamt 21 Items, bei denen die Zustimmung der Teilnehmenden auf einer vierstufigen Likert-Skala abgefragt wird $(1=$ stimme überhaupt nicht $\mathrm{zu} \ldots 4=$ stimme 


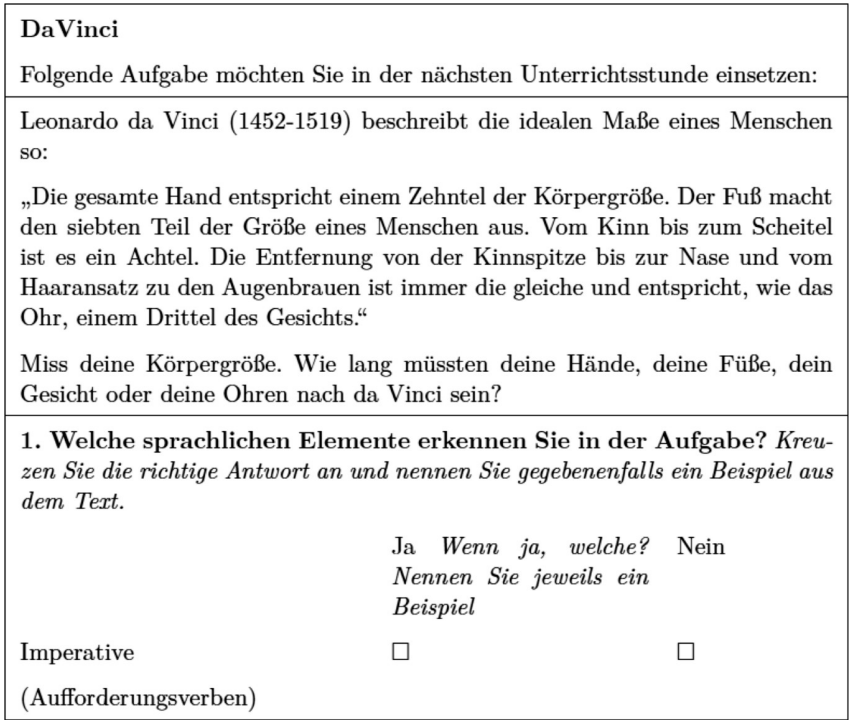

Abbildung 2: Beispielitem Paper-Pencil-Test (aus Ehmke et al. 2018)

völlig zu). Das Instrument gliedert sich in drei Teilskalen auf (vgl. Tabelle 1). Die Skala (1) Sprachgebrauch zu Hause erfasst epistemologische Überzeugungen der Probanden über den häuslichen Sprachgebrauch von migrationsbedingt mehrsprachigen SuS. Überzeugungen zu (2) Mehrsprachigkeit im Fachunterricht beziehen sich konkret auf die Gestaltung des Fachunterrichts in Bezug auf das Zulassen beziehungsweise die Unterdrückung von den Herkunftssprachen mehrsprachiger Lernender. Die Skala (3) Zuständigkeit für Sprachförderung im Fachunterricht beinhaltet Aussagen, die Aufschlüsse über das Rollenverständnis in Hinblick auf Sprachförderung im Fachunterricht zulassen.

\begin{tabular}{lrrr}
\hline Skala & Items & $\boldsymbol{\alpha}_{\mathfrak{t 1}}$ & $\boldsymbol{\alpha}_{{ }_{0}}$ \\
Gesamtskala & 21 & .72 & .79 \\
(1) Sprachgebrauch zu Hause & 5 & .78 & .77 \\
(2) Mehrsprachigkeit im Fachunterricht & 7 & .66 & .71 \\
(3) Zuständigkeit für Sprachförderung & 9 & .68 & .68 \\
\hline
\end{tabular}

Tabelle 1: Übersicht der eingesetzten Skalen zu Überzeugungen 


\subsection{Stichprobe}

Im Sommersemester 2017 nahmen insgesamt 43 Teilnehmende (31 weiblich; zwölf männlich) am WBS teil. Diese waren im Durchschnitt 37 Jahre alt und unterrichteten durchschnittlich bereits fünf bis neun Jahre. 34 Teilnehmende gaben Deutsch als ihre Erstsprache an.

23 der 40 Teilnehmenden nahmen an dem WBS für berufliche Schulen (Gruppe B) teil, welches im Sommersemester spezifisch für Lehrkräfte von vier beruflichen Schulen in NRW als In-House-Konzept angeboten wurde. Die anderen 17 Teilnehmenden nahmen an dem WBS für Lehrkräfte aller Schulformen (Gruppe A) teil, welches in den Räumlichkeiten der Universität Paderborn angeboten wurde.

\subsection{Methodisches Vorgehen}

Die Auswertung der Fragebögen zur Zufriedenheit erfolgte induktiv durch Kategorienbildung aus dem Datenmaterial. Für die Auswertung der Antworten auf geschlossene Fragen wurde für die Ermittlung von Gruppenunterschieden zwischen zwei unabhängigen Stichproben der Mann-Whitney-U-Test (Wilcoxon Rangsummen-Test) verwendet, der überprüft, ob die zentralen Tendenzen zweier unabhängiger Stichproben verschieden sind. Der Mann-Whitney-U-Test wird verwendet, wenn die Voraussetzungen für einen $t$-Test für unabhängige Stichproben nicht erfüllt sind. Ebenso eignet sich der Test besonders für kleine Stichproben.

Die Dateneingabe und -analyse des Paper-Pencil-Kompetenztests und des Fragebogens zu Überzeugungen erfolgte mit SPSS 25 (IBM 2017).

Der Kompetenztest wurde auf Basis des Rasch-Modells (Rost 2004) unter Einsatz des Programms ConQuest (Adams et al. 2012) skaliert. Fehlende Werte wurden als falsche Antworten gewertet. Die Item-Parameter der Normierungsstichprobe des DaZKom-Projekts wurden zur Ermittlung der Personenfähigkeitsschätzer (Weighted Likelihood Estimates - WLE) herangezogen. Um den Kompetenzzuwachs zu messen wurde die Effektstärke Cohen's d (Cohen 1988) berechnet und ein Test zur Ermittlung signifikanter Mittelwertunterschiede durchgeführt.

Aufgrund der kleinen Teilstichproben wurde zur Ermittlung signifikanter Unterschiede zwischen Messzeitpunkt ( $t$ ) 1 und 2 ebenfalls der Wilcoxon-Test eingesetzt (Schwarz 2016).

Für den Fragebogen zu Überzeugungen wurde ebenso vorgegangen. Um alle eingesetzten Items konsistent interpretieren zu können (z. B. hohe oder niedrige Ausprägung einer Skala), wurden negativ formulierte Items rekodiert. 


\section{Ergebnisse}

\subsection{Zufriedenheit}

(1) Wie zufrieden sind die Lebrkräfte mit dem WBS? Als wie relevant für die berufliche Praxis werden die Inhalte empfunden?

Die Betrachtung der Ergebnisse zur Zufriedenheit ergibt, dass 93\% aller Teilnehmenden den Aufbau des WBS als Blended-Learning-Angebot mit wöchentlichen Präsenzseminaren und zusätzlichen Workshops als gut bis sehr gut bewerten. Das inhaltliche Niveau betrachten 65\% der Lehrkräfte als angemessen, wobei 19\% es als hoch und $21 \%$ es als niedrig einschätzen. $88 \%$ aller Lehrkräfte würden den Studiengang weiterempfehlen. Die Atmosphäre in den Seminaren empfinden alle teilnehmenden Lehrkräfte als (sehr) angenehm. Die Erwartungen an das Studium haben sich bei 19\% der Lehrkräfte voll und ganz erfüllt. Diese geben als Begründung vor allem an, dass sie $»($ sehr) viel gelernt « und »einen anderen Blickwinkel zum Lernen erhalten haben «. Bei 61\% der Lehrkräfte haben sich die Erwartungen überwiegend erfüllt. Neben der positiven Begründung, viel gelernt zu haben, wird von diesen Lehrkräften Zeitdruck und Wiederholung bereits aus anderen Zusammenhängen bekannter Inhalte kritisiert sowie der Wunsch nach einem stärkeren Praxisbezug geäußert. 21\% der Teilnehmenden gaben an, dass sich ihre Erwartungen an das Studium eher nicht erfüllt haben. Diese Antwort wird mit dem Wunsch nach mehr Praxisbezug, mehr Informationen zur deutschen Sprache und mit aus Studium und Referendariat bekannten Inhalten begründet.

Der Vergleich der Antworten beider Gruppen ergibt bei den meisten Fragen keine signifikanten Unterschiede (vgl. Tabelle 2). Lediglich die Wahrnehmung des inhaltlichen Niveaus der Weiterbildung unterscheidet sich signifikant: Die Lehrkräfte der Gruppe A schätzen das inhaltliche Niveau der Weiterbildung im Vergleich zu den Teilnehmenden der Gruppe B als niedriger ein. Der Unterschied auf der Skala ist nach Cohen (1988) als klein bis mittel anzusehen.

\begin{tabular}{lrrrrrrr}
\hline & A & \multicolumn{7}{c}{ B } & & & & \\
& MW & SD & MW & SD & Z & p & $\mathbf{r}^{*}$ \\
Aufbau & 1.8 & 0.6 & 1.8 & 0.4 & -.52 & .604 & - \\
Inhaltliches Niveau & 3.2 & 0.6 & 2.8 & 0.5 & -2.04 & .041 & .32 \\
Erfüllung von Erwartungen & 2.2 & 0.6 & 1.9 & 0.6 & -1.25 & .210 & - \\
Atmosphäre & 1.2 & 0.4 & 1.3 & 0.5 & -.70 & .069 & - \\
Weiterempfehlung & 1.2 & 0.4 & 1.0 & 0.2 & -1.82 & .486 & - \\
\hline
\end{tabular}

Tabelle 2: Ergebnisse zur Zufriedenheit der Teilnehmenden mit der Weiterbildung 
Auf die offene Frage »Was hat Ihnen besonders gefallen? « nannten die Teilnehmenden von den vier Faktoren, die laut Lipowsky $(2010$, 52) die Zufriedenheit von Lehrkräften mit Fortbildungen beeinflussen, am häufigsten den Praxisbezug und die Möglichkeit zu Austausch und Partizipation. Häufig wurden auch einzelne Seminarinhalte sowie die »thematische Vielfalt«, »die Sensibilisierung für sprachliche Schwierigkeiten der DaZ-Lerner « und »die Vermittlung von fachlichen Neuigkeiten « angeführt. Darüber hinaus gibt es Nennungen wie »Struktur « und »Aufbau des Seminars «.

Als inhaltlich besonders relevant empfanden die Teilnehmenden vor allem die Themen »Diagnostik« und »Sprachförderung im Fach«. Die Inhalte der Workshops wurden vor allem von den Teilnehmern der B-Gruppe als relevant eingeschätzt. Was die inhaltliche Relevanz der Workshops »Traumapädagogik « und »Alphabetisierung « angeht, so schätzen insgesamt $70 \%$ der Lehrkräfte den Workshop Traumapädagogik und 78\% der Lehrkräfte den Workshop Alphabetisierung als (sehr) relevant.

\begin{tabular}{lrrrrrrr}
\hline & A & \multicolumn{1}{c}{ B } & & & & \\
& MW & SD & MW & SD & z & P & r* $^{*}$ \\
Traumapädagogik & 1.6 & 0.8 & 1.5 & 0.8 & -.13 & .900 & - \\
Alphabetisierung & 1.5 & 0.9 & 1.6 & 0.7 & -.61 & .542 & - \\
\hline
\end{tabular}

Tabelle 3: Ergebnisse zu empfundener inhaltlicher Relevanz der Themenbereiche »Traumapädagogik« und »Alphabetisierung"

Der Vergleich der Relevanz-Einschätzungen der Workshopteilnehmenden der beiden Gruppen zeigt keinen signifikanten Unterschied (vgl. Tabelle 3). Somit kann angenommen werden, dass die Lehrkräfte mit dem Weiterbildungsangebot zufrieden waren und es als relevant einschätzen.

\subsection{Kognition - Facette Wissen}

(2) Kann für die teilnehmenden Lehrkräfte des WBS ein Wissenszuwachs im Bereich Deutsch als Zweitsprache festgestellt werden?

Die Analyse hinsichtlich der Kompetenzentwicklung im Bereich Deutsch als Zweitsprache durch das WBS zeigt, dass sich generell ein signifikanter Wissenszuwachs zwischen den beiden Messzeitpunkten $\mathrm{t} 1$ und $\mathrm{t} 2$ abbilden lässt (vgl. Tabelle 4). Auf den Skalen Fachregister und Didaktik zeigt sich ebenso, dass die teilnehmenden Lehrkräfte signifikant dazulernen. Für die Subskala Mehrsprachigkeit ist kein Kompetenzzuwachs 
zu verzeichnen, allerdings starten die Teilnehmenden bei t 1 auch schon mit einem deutlich höheren Wert als auf den anderen beiden Teilskalen.

\begin{tabular}{lrrrrrr}
\hline & t1 & \multicolumn{1}{c}{ t2 } & & & \\
& M & SD & M & SD & d & P \\
Gesamtskala & -0.007 & 0.632 & 0.544 & 0.701 & 0.825 & $<.01$ \\
Subskala Fachregister & -0.076 & 0.936 & 0.694 & 0.951 & 0.816 & $<.01$ \\
Subskala Didaktik & -0.717 & 1.457 & 0.260 & 1.257 & 0.719 & $<.01$ \\
Subskala Mehrsprachigkeit & 0.427 & 0.520 & 0.518 & 0.575 & - & n.s. \\
\hline
\end{tabular}

Tabelle 4: Kompetenzentwicklung Gesamtstichprobe

Bei der Gegenüberstellung der beiden Gruppen A und B zeigen sich Unterschiede, sowohl auf der Gesamtskala als auch auf den Subskalen (vgl. Tabelle 5). Während die Teilnehmenden der Gruppe A weder auf der Gesamtskala noch auf einer der drei Subskalen einen Kompetenzzuwachs zeigen, lässt sich für die Gruppe B ein signifikanter Kompetenzzuwachs sowohl auf der Gesamtskala als auch auf den Subskalen Fachregister und Didaktik verzeichnen. Für die Subskala Mehrsprachigkeit zeigt sich für beide Gruppen kein signifikanter Zuwachs.

\begin{tabular}{|c|c|c|c|c|c|c|}
\hline & t1 & & t2 & & & \\
\hline & $M$ & SD & $M$ & SD & $z$-Wert & $\mathbf{P}$ \\
\hline \multicolumn{7}{|c|}{ Gesamtskala } \\
\hline Gruppe A & 0,127 & 0,494 & 0,394 & 0,798 & $-1,444$ & n.s. \\
\hline Gruppe B & $-0,106$ & 0,712 & 0,655 & 0,614 & $-4,107$ & $<.01$ \\
\hline \multicolumn{7}{|c|}{ Subskala Fachregister } \\
\hline Gruppe A & 0,055 & 0,671 & 0,505 & 1,066 & $-1,728$ & n.s. \\
\hline Gruppe B & $-0,172$ & 1,096 & 0,833 & 0,853 & $-3,833$ & $<.01$ \\
\hline \multicolumn{7}{|c|}{ Subskala Didaktik } \\
\hline Gruppe A & $-0,551$ & 1,396 & 0,160 & 1,611 & $-1,444$ & n.s. \\
\hline Gruppe B & $-0,840$ & 1,519 & 0,334 & 0,949 & $-3,620$ & $<.01$ \\
\hline \multicolumn{7}{|c|}{ Subskala Mehrsprachigkeit } \\
\hline Gruppe A & 0,573 & 0,413 & 0,314 & 0,537 & $-1,681$ & n.s. \\
\hline Gruppe B & 0,320 & 0,572 & 0,669 & 0,566 & $-2,281$ & n.s. \\
\hline
\end{tabular}

Tabelle 5: Kompetenzentwicklung Gruppen A und B 
Beim Blick auf die Entwicklung der Kompetenzstufen zeigt sich, dass, ähnlich wie bei der Stichprobe des Standard-Settings, sich zum Messzeitpunkt t 1 mehr als $90 \%$ aller am WBS teilnehmenden Lehrkräfte lediglich auf der Stufe »Unter-Mindest-Standard « befinden (vgl. Tabelle 6). Drei Personen befinden sich auf dem »Mindest-Standard « und niemand der 40 Teilnehmer ist auf dem $\gg$ Regel-Standard « zu finden. Nach der Teilnahme am WBS schaffen es 20\% den »Mindest-Standard « und sogar 12,5\%, also fünf Lehrkräfte, den »Regel-Standard « zu erreichen.

Ein Vergleich der beiden Gruppen A und B zeigt auch an dieser Stelle, dass die Lehrkräfte in der Gruppe B insgesamt deutlich bessere Ergebnisse erzielen, jedoch werden die verhältnismäßig schlechten Ergebnisse der Teilnehmenden in der Gruppe A etwas relativiert, da immerhin mehr als 40\% (sieben Personen) den Mindest- oder sogar Regel-Standard erreichen.

Betrachtet man die Gesamtgruppe, kann also für die teilnehmenden Lehrkräfte des WBS ein Wissenszuwachs im Bereich Deutsch als Zweitsprache festgestellt werden.

\begin{tabular}{|c|c|c|c|c|c|c|c|}
\hline & t1 & & & t2 & & & \\
\hline & Gesamt & A & B & Gesamt & A & B & $\begin{array}{l}\text { Standard- } \\
\text { setting } \\
(n=1383)\end{array}$ \\
\hline Regel-Standard & 0,0 & 0,0 & 0,0 & $\begin{array}{r}12,5 \\
(5)\end{array}$ & $\begin{array}{l}5,9 \\
(1)\end{array}$ & $\begin{array}{r}17,4 \\
(4)\end{array}$ & 1,5 \\
\hline Mindest-Standard & $\begin{array}{l}7,5 \\
(3)\end{array}$ & $\begin{array}{l}5,9 \\
(1)\end{array}$ & $\begin{array}{l}8,7 \\
(2)\end{array}$ & $\begin{array}{r}20,0 \\
(8)\end{array}$ & $\begin{array}{r}35,3 \\
(6)\end{array}$ & $\begin{array}{l}8,7 \\
(2)\end{array}$ & 7,2 \\
\hline $\begin{array}{l}\text { Unter-Mindest- } \\
\text { Standard }\end{array}$ & $\begin{array}{l}92,5 \\
(37)\end{array}$ & $\begin{array}{l}94,7 \\
(16)\end{array}$ & $\begin{array}{l}91,3 \\
(21)\end{array}$ & $\begin{array}{l}67,5 \\
(27)\end{array}$ & $\begin{array}{l}58,8 \\
(10)\end{array}$ & $\begin{array}{l}73,9 \\
(17)\end{array}$ & 91,3 \\
\hline
\end{tabular}

Tabelle 6: Kompetenzstufenwerte in \%

\subsection{Kognition - Facette Überzeugungen/Beliefs}

(3) Kann für die teilnehmenden Lehrkräfte des WBS einepositive Veränderung der Überzeugungen zu sprachlich-kultureller Heterogenität in Schule und Unterricht festgestellt werden?

Die Analyse der Befragung zu den Überzeugungen hinsichtlich sprachlich-kultureller Heterogenität (vgl. Tabelle 7) zeigt, dass das WBS einen positiven Effekt auf die Überzeugungen der teilnehmenden Lehrkräfte hat. Generell lässt sich festhalten, dass die Lehrkräfte nach der Teilnahme signifikant positivere Überzeugungen als zu Beginn der Fortbildung zeigen. Für die Gesamtheit der Stichprobe lässt sich auf der Gesamtskala nach Cohen (1988) ein großer Effekt nachweisen $(r=0,55)$, der nach Hattie (2008) in der sogenannten $\gg$ zone of desired effects « liegt. In Hinblick auf die Teilskalen lassen 
sich ebenfalls signifikante Veränderungen für die Skalen (1) Sprachgebrauch zu Hause und (2) Mehrsprachigkeit im Fachunterricht belegen. Keine signifikante Veränderung zeigt jedoch die Skala (3) Zuständigkeit für Sprachförderung.

Betrachtet man die Ergebnisse für die beiden Gruppen A und B einzeln, wird deutlich, dass sich die Überzeugungen der Teilnehmenden am Standort A nicht signifikant verändert haben. Weder auf der Gesamtskala noch auf den drei Teilskalen lassen sich bedeutsame Effekte nachweisen. Der Standort B hingegen zeigt durchgehend Veränderungen bei den Überzeugungen der Lehrkräfte. Die größte Entwicklung zeigt sich für die Skala (2) Mehrsprachigkeit im Fachunterricht, gefolgt von Skala (1) Sprachgebrauch $z u$ Hause. Anders als für die Gesamtstichprobe und die Stichprobe des Standorts A kann für den Standort B auch eine signifikante Veränderung der Überzeugungen zur Skala (3) Zuständigkeit für Sprachförderung festgestellt werden. Die Veränderungen auf allen (Teil-)Skalen sind für die Teilstichprobe des Standorts B nach Cohen (1988) als große Effekte einzuordnen und befinden sich angelehnt an Hattie (2008) in der » zone of desired effects «.

\begin{tabular}{|c|c|c|c|c|c|c|c|}
\hline & T1 & & T2 & & & & \\
\hline & MW & SD & MW & SD & $z$ & $\mathbf{p}$ & $\mathbf{r}^{*}$ \\
\hline \multicolumn{8}{|l|}{ Gesamtskala } \\
\hline Gesamtstichprobe $(n=35)$ & 3.1 & 0.3 & 3.3 & 0.3 & -3.24 & .001 & 0.55 \\
\hline Gruppe $A(n=15)$ & 3.2 & 0.3 & 3.2 & 0.3 & -0.60 & .552 & - \\
\hline Gruppe B $(n=20)$ & 3.0 & 0.3 & 3.5 & 0.3 & -3.92 & .000 & 0.88 \\
\hline \multicolumn{8}{|c|}{ (1) Sprachgebrauch zu Hause } \\
\hline Gesamtstichprobe & 2.7 & 0.6 & 3.2 & 0.6 & -3.61 & .000 & 0.61 \\
\hline Gruppe A & 2.9 & 0.6 & 3.1 & 0.6 & -1.79 & .073 & - \\
\hline Gruppe B & 2.7 & 0.7 & 3.3 & 0.6 & -3.13 & .002 & 0.70 \\
\hline \multicolumn{8}{|c|}{ (2) Mehrsprachigkeit im Fachunterricht } \\
\hline Gesamtstichprobe & 3.1 & 0.4 & 3.3 & 0.4 & -2.44 & .015 & 0.41 \\
\hline Gruppe A & 3.2 & 0.4 & 3.1 & 0.4 & -0.63 & .528 & - \\
\hline Gruppe B & 3.0 & 0.4 & 3.4 & 0.4 & -3.63 & .000 & 0.81 \\
\hline \multicolumn{8}{|c|}{ (3) Zuständigkeit für Sprachförderung } \\
\hline Gesamtstichprobe & 3.3 & 0.4 & 3.4 & 0.4 & -1.37 & .169 & - \\
\hline Gruppe A & 3.4 & 0.3 & 3.2 & 0.4 & -1.04 & .297 & - \\
\hline Gruppe B & 3.3 & 0.4 & 3.6 & 0.3 & -2.59 & .010 & 0.58 \\
\hline
\end{tabular}

Tabelle 7: Überzeugungen - Mittelwertsvergleich 


\section{$5 \quad$ Zusammenfassung und Diskussion}

Zusammenfassend lässt sich festhalten, dass das WBS insgesamt eine sehr positive Wirkung bei den teilnehmenden Lehrkräften hat. Sowohl die Zufriedenheit mit der Konzeption des WBS und der damit eng verbundenen Inhalte als auch die Entwicklung der DaZ-Kompetenzen und der Überzeugungen über die Laufzeit des WBS hinweg sind durchweg als sehr positiv zu bewerten. Das WBS kann somit einerseits als Sensibilisierung andererseits aber auch als Vorbereitung für ein inklusive(re)s Lehrerhandeln gesehen werden. Die verschiedenen Weiterbildungsinhalte zielen auf einen multiperspektivischen Ansatz von DaZ ab, der die verschiedenen Facetten von Sprache und migrationsbedingter Mehrsprachigkeit ganzheitlich in den Blick nimmt.

In Hinblick auf die Ebene Reaktionen und Einschätzungen der teilnehmenden Lehrpersonen (1) fällt besonders auf, dass die Teilnehmenden überwiegend zufrieden mit den Inhalten sind und diese auch als relevant erachten. Als ein Faktor für Unzufriedenheit wird von einigen/wenigen Teilnehmenden der fehlende Praxisbezug angeführt, wobei auf die Frage > Was hat Ihnen an der Fortbildung besonders gefallen?< gerade der Praxisbezug neben der Möglichkeit zum Austausch von den anderen Teilnehmenden am häufigsten genannt wird. Dies ist auch in anderen Evaluationen sowohl im Hochschulbereich (Wenzl et al. 2017) als auch im Weiterbildungsbereich (Altrichter 2017) zu beobachten. Dieser Widerspruch lässt sich möglicherweise mit unterschiedlichen Vorstellungen der Teilnehmenden zum Praxisbezug einer Fortbildung erklären, was sich aber anhand der vorliegenden Daten nicht untersuchen lässt.

Auf der Ebene Erweiterung der Lehrerkognitionen (2) können statistisch signifikante Veränderungen auf den Skalen zu DaZ-Kompetenzen als auch zu Überzeugungen beobachtet werden. Hinsichtlich des Wissens fällt auf, dass auf der Subskala Mehrsprachigkeit keine signifikante Veränderung stattfindet. Dies könnte damit erklärt werden, dass die Teilnehmenden bereits mit sehr hohen Werten starten, was wiederum auf die schon vorhandene Sensibilität zurückzuführen sein könnte, die es braucht, um an einer Weiterbildung in diesem Bereich interessiert zu sein. Weiterhin umfasst die Dimension Mehrsprachigkeit im DaZKom-Modell den Bereich Zweitspracherwerbstheorien, der in den Inhalten des WBS nicht berücksichtigt wurde (vgl. hierzu auch Böttger et al. 2020). Ein ähnliches Bild zeigt sich für die Subskala Zuständigkeit für Sprachförderung im Bereich der Überzeugungen. Hier lässt sich für die Gesamtstichprobe keine positive Entwicklung über die Seminarlaufzeit hinweg feststellen. Eine wahrscheinliche Ursache hierfür ist - wie auch für die Dimension Mehrsprachigkeit -, dass Lehrkräfte, die neben ihrem Beruf an einem zeitlich sehr aufwendigen und umfangreichen WBS teilnehmen, bereits vor ihrer Teilnahme von der eigenen Zuständigkeit für Sprachbildung überzeugt sind.

Beim Vergleich der beiden Gruppen zeigen sich vor allem auf der Ebene 2 (Erweiterung der Lehrerkognitionen) große Unterschiede. Während Standort B sowohl auf den 
Gesamtskalen der Kompetenz- und Überzeugungsuntersuchung als auch auf jeweils zwei der drei Subskalen statistisch signifikante Zuwächse verzeichnen kann, bleibt bei Standort A sowohl ein statistisch signifikanter Kompetenzzuwachs als auch eine positive Veränderung der Überzeugungen aus. Dieses Ergebnis ist insofern zu diskutieren, als dass sich in sämtlichen weiteren Untersuchungen zur DaZ-Kompetenzentwicklung der Kompetenzzuwachs und die Einstellungen immer auf statistisch signifikante Art und Weise verändern (Paetsch et al. 2019; Böttger et al. 2020; Fischer und Lahmann in Begutachtung; Hammer und Berkel-Otto 2019). In diesem Fall ist die Nicht-Veränderung besonders deswegen interessant, da beide Standorte dieselben Inhalte von denselben Dozierenden erhalten haben. Eine mögliche Erklärung für diese sehr deutlichen Unterschiede könnte sein, dass die Zusammensetzung der Gruppen sehr unterschiedlich aussah. Während Standort B von Kollegen vier räumlich eng benachbarter Schulen absolviert wurde, die Teilnehmenden sich also bereits vorher teilweise untereinander kannten, setzte sich die Gruppe am Standort A aus Lehrkräften verschiedener Schulen und Schulformen zusammen. Die Mitglieder dieser Gruppe kannten sich vorher überwiegend nicht. Somit hatten die Teilnehmenden des Standorts B die Möglichkeit, sich auch während des Lehrbetriebs über Inhalte oder Fragen bzgl. des WBS auszutauschen, während Lehrkräfte des Standorts A überwiegend auf sich allein gestellt waren. Positive Effekte auf Weiterbildungsmaßnahmen durch kollegialen Austausch konnten in anderen Studien bereits gezeigt werden (Altrichter 2017; Holtappels 2014). Zudem gibt es Hinweise darauf, dass die Teilnehmenden des Standorts B in Bezug auf ihre Teilnahme am WBS intensiv durch ihre Schulleitungen unterstützt und wertgeschätzt wurden. Das kann vor allem damit erklärt werden, dass die Initiative zur Weiterbildung am Standort B von den Schulen selbst ausging, sie von Beginn an also eine klare Zielsetzung (Vorbereitung auf die vielen neu Zugewanderten durch das Fit für mehrProgramm) mit der Teilnahme am WBS verfolgten, während bei den Lehrkräften des Standorts A möglicherweise viel unspezifischere bzw. unterschiedlichere Zielsetzungen (Karrierechancen verbessern o. Ä.) verfolgt wurden.

Weiterhin ist es interessant, dass Lehrkräfte des Standorts A das inhaltliche Niveau der Weiterbildung niedriger einschätzen als diejenigen des Standorts B. Eine mögliche Ursache für die unterschiedliche Einschätzung könnte sein, dass Lehrkräfte an Berufskollegs häufig eine ganz andere Ausbildung erhalten (haben), in der die Themen DaZ und Sprachbildung eine noch untergeordnete Rolle einnehmen. Das WBS wird daher von ihnen ggf. als inhaltlich anspruchsvoller eingeschätzt.

Zusammen mit den Ergebnissen auf der Kognitionsebene könnte eine ähnliche Begründung wie in Lipowsky und Rzejak (2012) herangezogen werden:

»Eine aktuelle Studie mit Grundschullehrpersonen ergab, dass die von den Lehrpersonen eingeschätzte persönliche Relevanz der Fortbildung mit deren Einschätzung der 
Fortbildung als Herausforderung einherging, welche wiederum die selbsteingeschätzte Verarbeitung der Fortbildungsinhalte und hierüber auch Veränderungen in Überzeugungen vorhersagen konnte « (Böhmer, Kunter und Hertel 2011).

\section{Ausblick}

Der Beitrag macht deutlich, dass ein umfassendes Konzept vonnöten ist, um Kompetenzen im Bereich DaZ und Sprachbildung einerseits zu erforschen und ihnen andererseits eine zentrale Rolle in allen drei Phasen der Lehrkräftebildung und hier vor allem in der dritten Phase einzuräumen. Dabei ist allerdings speziell für den Bereich DaZ und Sprachbildung insgesamt noch zu wenig über die Beschaffenheit von Lerngelegenheiten bekannt, weil die Aus- und Weiterbildung sprach- und kultursensibler und somit inklusiv agierender Lehrkräfte noch wenig erforscht ist. Dieser Beitrag schafft es, diese Lücke ein Stück weit zu schließen. Die Erarbeitung eines möglichen Curriculums, zum Beispiel auf Basis des DaZKom-Kompetenzmodells, für umfangreichere Aus- und Weiterbildungsmaßnahmen für Lehrkräfte steht nach wie vor aus.

Die Ergebnisse zeigen darüber hinaus, dass eine Weiterbildung im Umfang von sechs CP, den viele Module zum Themenbereich DaZ und Sprachbildung aufweisen, zwar für einen geringen Teil (12,5\%) der Lehrkräfte ausreicht, um den Regelstandard des DaZKom-Modells zu erreichen, dass aber die Mehrzahl der Lehrkräfte ein umfangreicheres Training benötigt, um diesen Standard zu erreichen. Somit muss die Frage gestellt werden, ob und wie die DaZ-Lehrkräfteaus- und -weiterbildung in Zukunft im Sinne einer »Theorie des sprachlichen und literarischen Lehrens und Lernens in heterogenen Lerngruppen [...], die sich auf alle LernerInnen unabhängig von ihren sprachlichen, kognitiven, körperlichen, sozialen, migrationsbezogenen und emotionalaffektiven Voraussetzungen bezieht und die auf empirischer Grundlage die Ziele, Bedingungen, Prozesse und Resultate sprachlicher Bildung beschreibt « (Becker-Mrotzek und Linnemann 2017, 111) ausgestaltet sein kann. Zudem könnte beforscht werden, welche Faktoren dazu führen, dass 12,5 Prozent der Teilnehmenden den Regelstandard erreicht haben bzw. inwiefern sich die Ausgangslage dieser Teilnehmenden von denen der anderen unterscheidet oder was dazu führt, dass diese Teilnehmenden die Inhalte des WBS erfolgreicher aufnehmen/verarbeiten.

Die Ergebnisse zeigen des Weiteren, dass es sich bei der Ausgestaltung des WBS im Sinne des Didaktischen Modells für inklusives Lehren und Lernen (DiMiLL) um eine geeignete Maßnahme handelt, in der Lehrkräfte für verschiedene Facetten von Inklusion sensibilisiert werden.

Wie bereits erwähnt, zeigt sich, dass der Faktor Praxisbezug entscheidend für die Zufriedenheit ist und darüber hinaus auch Einfluss auf andere Ebenen hat. So zeigt 
Massumi 2016, dass eine durch Theorie-Praxis-Verzahnung gekennzeichnete Lerngelegenheit im Bereich der Professionalisierung angehender Lehrkräfte für das Unterrichten in der Migrationsgesellschaft den individuellen und professionellen Entwicklungsprozess der Teilnehmenden beeinflusst und sich insbesondere auch auf Überzeugungen und subjektive Theorien auswirkt. Mavruk 2018 zeigt ebenfalls, dass Lerngelegenheiten, die durch Theorie-Praxis-Verzahnung gekennzeichnet sind, zu veränderten Überzeugungen in Bezug auf die Notwendigkeit und Bedeutung sprachbildenden Unterrichts und $\mathrm{zu} \gg \mathrm{DaZ}$-spezifischen Handlungsorientierungen « (295) führen können. Blumberg et al. 2019 zeigen durch Theorie-Praxis-Verzahnung begründete Veränderungen in der Selbstwirksamkeitserwartung bezüglich der Planung und Durchführung sprachbildenden Fachunterrichts. Diese Erkenntnisse gaben Anlass für die Umkonzeptionierung des WBS dahingehend, dass nun eine stärkere Verzahnung der fachwissenschaftlichen und fachdidaktischen Ausbildung im Bereich DaZ und Sprachbildung mit Praxiserfahrungen erfolgt.

Zukünftige Evaluationsstudien sollten die Zusammenhänge qualitativer und quantitativer Daten stärker in den Fokus rücken und der Frage nachgehen, ob und inwieweit die den eingesetzten Testinstrumenten zugrundeliegenden Konstrukte Teil der Seminarkonzeption sind.

\section{Anmerkungen}

1 Siehe hierzu https://www.behindertenrechtskonvention.info/inklusion-3693/ (24.01.2019).

2 In Deutschland liegen die Verantwortlichkeiten für die Lehreraus- und -weiterbildung in den Händen der jeweiligen Bundesländer.

3 Nach dem European Credit Transfer System (ECTS) entspricht ein Credit Point (CP) oder Leistungspunkt (LP) einem ungefähren Arbeitsaufwand von 30 Stunden.

4 Kennwerte zur Dimensionalität und psychometrischen Qualität des Instruments sind in Fischer und Ehmke (2019) zu finden.

\section{Literatur}

Adams, Raymond, Margaret Wu und Mark Wilson. 2012. ACER ConQuest. Australian Council for Educational Research (ACER) [Computer Software].

Altrichter, Herbert. 2017. „Lehrerfortbildung, die einen Unterschied macht.« Schwerpunktheft Weiterbildung für Lehrer, 12-15.

Baumann, Barbara. 2017. "Sprachförderung und Deutsch als Zweitsprache in der Lehrerbildung - ein deutschlandweiter Überblick." In Deutsch als Zweitsprache in der Lehrerbildung, herausgegeben von Michael Becker-Mrotzek, Peter Rosenberg, Christoph Schroeder und Annika Witte, 9-26. Münster: Waxmann.

Baumert, Jürgen, und Mareike Kunter. 2006. „Stichwort: Professionelle Kompetenz von Lehrkräften.« Zeitschrift für Erziehungswissenschaft 9: 469-520. 
Becker-Mrotzek, Michael, und Markus Linnemann. 2017. »lnklusive Fachdidaktik Deutsch. In Lexikon Inklusion. herausgegeben von Kerstin Ziemen, 111-12. Göttingen: Vandenhoeck \& Ruprecht.

Becker-Mrotzek, Michael, und Hans-Joachim Roth. 2017. Sprachliche Bildung - Grundlagen und Handlungsfelder. Münster: Waxmann.

Becker-Mrotzek, Michael, und Hans-Joachim Roth. 2017. "Sprachliche Bildung - Grundlagen und Konzepte. «In Sprachliche Bildung - Grundlagen und Handlungsfelder, herausgegeben von Michael Becker-Mrotzek und Hans-Joachim Roth. 11-36. Münster: Waxmann.

Biewer, Gottfried, Michelle Proyer und Getraud Kremsner. 2019. Inklusive Schule und Vielfalt. Stuttgart: Kohlhammer.

Blömeke, Sigrid, Jan-Eric Gustafsson und Richard J. Shavelson. 2015. „Beyond dichotomies: Competence viewed as a continuum. Zeitschrift für Psychologie 223: 3-13. https://doi.org/10. 1027/2151-2604/a000194

Blömeke, Sigrid, Gabriele Kaiser und Rainer Lehmann. 2008. Professionelle Kompetenz angehender Lehrerinnen und Lehrer: Wissen, Überzeugungen und Lerngelegenheiten deutscher Mathematikstudierender und -referendare; erste Ergebnisse zur Wirksamkeit der Lehrerausbildung. Münster: Waxmann.

Blömeke, Sigrid, und Fritz Oser. 2012. »Überzeugungen von Lehrpersonen. Einführung in den Thementeil.« Zeitschrift für Pädagogik, 58 (4): 415-21.

Blumberg, Eva, Constanze Niederhaus, Timm Albers und Amra Havkic. 2019. »Durchgängige Sprachbildung und Inklusion in der sachunterrichtsdidaktischen Lehrer*innenbildung - Eine interdisziplinäre Evaluationsstudie mit Sachunterrichtsstudierenden. In Inklusion im Sachunterricht, herausgegeben von Detlef Pech, Claudia Schomaker und Toni Simon. 169-81. Bad Heilbrunn: Klinkhardt.

Böhmer, Anna-Theresia, Mareike Kunter und Silke Hertel. 2011. Veränderungsbereitschaft von Lehrkräften - Empirische Überprüfung eines kognitiv-affektiven Modells der Überzeugungsveränderungen. Vortrag auf der Fachgruppentagung Pädagogische Psychologie der DPGs 14.-16.09.2011 in Erfurt.

Böttger, Lydia, Olga Groh, Lisa Otto, Nele Fischer, Svenja Hammer, Ulla Jung und Constanze Niederhaus. 2020. »Kompetenzzuwachs und veränderte Unterrichtspraxis. Erste Ergebnisse aus der Evaluation eines DaZ-Weiterbildungsstudiums für Lehrkräfte an beruflichen Schulen.« In Berufliche Integration durch Sprache, herausgegeben von Thomas Hochleitner und Jörg Roche. 167-85. Leverkusen: Verlag Barbara Budrich.

Bromme, Rainer. 1997. »Kompetenzen, Funktionen und unterrichtliches Handeln des Lehrers.«In Enzyklopädie der Psychologie: Themenbereich D Praxisgebiete. Serie I Pädagogische Psychologie. Bd. 3: Psychologie des Unterrichts und der Schule, herausgegeben von Franz E. Weinert. 177-212. Göttingen: Hogrefe.

Carlson, Sonja A. und Désirée Präg. 2018. »Der Prozess der Aufgabenentwicklung im DaZKomProjekt: von der Rahmenkonzeption bis zur Pilotierung des Testinstruments."In Professionelle Kompetenzen angehender Lehrkräfte im Bereich Deutsch als Zweitsprache, herausgegeben von Timo Ehmke, Svenja Hammer, Anne Köker, Udo Ohm und Barbara Koch-Priewe. 93-108. Münster: Waxmann.

Cohen, Jacob. 1988. Statistical power analysis for the behavioral sciences. Hillsdale: Erlbaum.

Ehmke, Timo, Svenja Hammer, Anne Köker, Udo Ohm und Barbara Koch-Priewe. 2018. Professionelle Kompetenzen angehender Lehrkräfte im Bereich Deutsch als Zweitsprache. Münster: Waxmann.

Fischer, Nele. 2018. »Professionelle Überzeugungen von Lehrkräften: vom allgemeinen Konstrukt zum speziellen Fall von sprachlich-kultureller Heterogenität in Schule und Unterricht."Psychologie in Erziehung und Unterricht, 65 (1): 35-51. 
Fischer, Nele, und Timo Ehmke. 2019. »Empirische Erfassung eines `messy constructsı: Überzeugungen angehender Lehrkräfte zu sprachlich-kultureller Heterogenität in Schule und Unterricht.« Zeitschrift für Erziehungswissenschaft, 22: 411-33.

Fischer, Nele, Svenja Hammer und Timo Ehmke. 2018. Überzeugungen zu Sprache im Fachunterricht: Erhebungsinstrument und Skalendokumentation. In Professionelle Kompetenzen angehender Lehrkräfte im Bereich Deutsch als Zweitsprache, herausgegeben von Timo Ehmke, Svenja Hammer, Anne Köker, Udo Ohm und Barbara Koch-Priewe. 149-84. Münster: Waxmann.

Gogolin, Ingrid. 2008. Der monolinguale Habitus der multilingualen Schule. Münster: Waxmann.

Goldschmidt, Pete, und Geoffrey Phelps. 2007. „Does teacher professional development affect content and pedagogical knowledge: How much and for how long?« National Center for Research on Evaluation, Standards and Student Testing (CRESST), Center for the Study of Evaluation (CSE). Los Angeles.

Gültekin-Karakoç, Nazan. 2018. „Sicherung der Inhaltsvalidität und Festlegung von Kompetenzstufen durch Expertenbefragungen. «In Professionelle Kompetenzen angehender Lehrkräfte im Bereich Deutsch als Zweitsprache, herausgegeben von Timo Ehmke, Svenja Hammer, Anne Köker, Udo Ohm und Barbara Koch-Priewe. 109-28. Münster: Waxmann.

Hachfeld, Anja, Sascha Schroeder, Yvonne Anders, Adam Hahn und Mareike Kunter. 2012. »Multikulturelle Überzeugungen - Herkunft oder Überzeugung? Welche Rolle spielen der Migrationshintergrund und multikulturelle Überzeugungen für das Unterrichten von Kindern mit Migrationshintergrund.« Zeitschrift für Pädagogische Psychologie, 26 (2): 101-20.

Hagemann, Vera, und Annette Kluge. 2014. »Einflussfaktoren auf den Erfolg von und Methoden der Erfolgsmessung beruflicher Weiterbildung."Wirtschaftspsychologie, 16 (2): 81-93.

Hallitzky, Maria, und Martina Schliessleder. 2008. »Welche pädagogischen Leitbilder haben Lehramtsstudierende in Bezug auf den Umgang mit migrationsbedingter Heterogenität?« In Chancenungleichheit in der Grundschule. Ursachen und Wege aus der Krise, herausgegeben von Jörg Ramseger und Matthea Wagener. 267-70. Wiesbaden: VS Verlag für Sozialwissenschaften.

Hammer, Svenja und Lisa Berkel-Otto. 2019. »Differing Teaching Formats: pre-service teachers' professional competency development in linguistically responsive teaching." Open Education Studies, 1 (1): 245-56. https://doi.org/10.1515/edu-2019-0018

Hammer, Svenja, Sonja A. Carlson, Timo Ehmke, Barbara Koch-Priewe, Anne Köker, Udo Ohm, Sonja Rosenbrock und Nina Schulze. 2015. "Kompetenz von Lehramtsstudierenden in Deutsch als Zweitsprache. Validierung des GSL-Testinstruments.« Zeitschrift für Pädagogik, 61:32-54.

Hammer, Svenja und Timo Ehmke. 2018. „Ergebnisse einer Validierungsstudie zum DaZKomTestinstrument." In Professionelle Kompetenzen angehender Lehrkräfte im Bereich Deutsch als Zweitsprache, herausgegeben von Timo Ehmke, Svenja Hammer, Anne Köker, Udo Ohm und Barbara Koch-Priewe. 185-99. Münster: Waxmann.

Hammer, Svenja, Nele Fischer und Barbara Koch-Priewe. 2016. „Überzeugungen von Lehramtsstudierenden zu Mehrsprachigkeit in der Schule.» In DDS - Die Deutsche Schule: Zeitschrift für Erziehungswissenschaft, Bildungspolitik und pädagogische Praxis, Beiheft 13: 149-74.

Hartwig, Svenja Janina, Franziska Schwabe, Miriam Marleen Gebauer und Nele McElvany. 2017. „Wie beurteilen Lehrkräfte und Lehramtsstudierende Leistungsheterogenität? Ausprägungen, Zusammenhänge und Prädiktoren von Einstellungen und Motivation.« Psychologie in Erziehung und Unterricht, 64 (2): 94-108.

Hattie, John. 2008. Visible Learning. London: Routledge.

Hochholdinger, Sabine, und Niclas Schaper. 2007. »Trainingsevaluation und Transfersicherung.« In 
Handbuch der Arbeits- und Organisationspsychologie, herausgegeben von Heinz Schuler und Karlheinz Sonntag. 625-33. Göttingen et al.: Hogrefe.

Holtappels, Heinz Günter. 2014. Schulentwicklung und Schulwirksamkeit als Forschungsfeld: Theorieansätze und Forschungserkenntnisse zum schulischen Wandel. Münster: Waxmann.

Klieme, Eckhard, Cordula Artelt, Johannes Hartig, Nina Jude, Olaf Köller, Manfred Prenzel und Wolfgang Schneider. 2010. PISA 2009: Bilanz nach einem Jahrzehnt. Münster: Waxmann.

Krumm, Hans-Jürgen. 2017. »Sprachliche Heterogenität - Verschiedenheit und gemeinsames Lernen. "In Inklusion, Diversität und das Lehren und Lernen fremder Sprachen, herausgegeben von Eva Burwitz-Melzer, Frank G. Königs, Claudia Riemer und Lars Schmelter. 145-57. Tübingen: Narr.

Lipowsky, Frank 2006. »Auf den Lehrer kommt es an.« Zeitschrift für Pädagogik, 51: 47-65.

Lipowsky, Frank 2010. »Lernen im Beruf.«In Lehrerinnen und Lehrer lernen. Konzepte und Befunde zur Lehrerfortbildung, herausgegeben von Florian H. Müller, Astrid Eichenberger, Manfred Lüders und Johannes Mayr. 51-72. Münster: Waxmann.

Lipowsky, Frank. 2014. »Theoretische Perspektiven und empirische Befunde zur Wirksamkeit von Lehrerfort- und -weiterbildung." In Handbuch der Forschung zum Lehrerberuf, herausgegeben von Ewald Terhart, Hedda Bennewitz und Martin Rothland. 511-41. Münster: Waxmann.

Lipowsky, Frank und Daniela Rzejak. 2012. »Lehrerinnen und Lehrer als Lerner - Wann gelingt der Rollentausch? Merkmale und Wirkungen wirksamer Lehrerfortbildungen.« Schulpädagogik heute, 3 (5): 1-17.

Lucas, Tamara, Ana Maria Villegas und Margaret Freedson-Gonzalez. 2008. »Linguistically responsive teacher education: Preparing classroom teachers to teach English language learners." Journal of Teacher Education, 59 (4): 361-73.

Lütke, Beate. 2017. „Deutsch als Zweitsprache-Module im Lehramtsstudium: Entwicklung, Relevanz und curriculare Konzepte.« FLuL-Fremdsprachen Lehren und Lernen, 46 (1): 27-42.

Maak, Diana, Julia Ricart Brede und Sebastian Born. 2015. »Einstellungen von Lehramtsstudierenden zu Mehrsprachigkeit erheben. Bericht über die Anlage eines Forschungsprojektes.« In Deutsch als Zweitsprache - Erwerb und Didaktik, herausgegebn von Heidi Rösch und Julia Webersik. 263-82. Stuttgart: Fillibach bei Klett.

Massumi, Mona. 2016. "Sprachförderung für geflüchtete Kinder und Jugendliche ohne Schulzugang." In Neu zugewanderte Schülerinnen und Schüler, herausgegeben von Claudia Benholz, Magnus Franz und Constanze Niederhaus. 197-216. Münster: Waxmann.

Mavruk, Gülsah. 2018. Microteaching in der universitären Lehrerausbildung: Rekonstruktion studentischer Erfahrungsräume im Berufsfeldpraktikum im Bereich Deutsch als Zweitsprache. Münster: Waxmann.

Nimz, Katharina, Sarah-Larissa Hecker und Anne Köker (2018). „Videobasierte Messung von DaZKompetenz bei Lehrkräften. «In Sprache im Unterricht. Ansätze, Konzepte und Methoden, herausgegeben von Celestine Caruso, Judith Hofmann, Andreas Rohde und Kim Schick. 439-52. Trier: WVT.

Paetsch, Jennifer, Annkathrin Darsow, Fränze Sophie Wagner, Svenja Hammer und Timo Ehmke. 2019. »Prädiktoren des Kompetenzzuwachses im Bereich Deutsch als Zweitsprache bei Lehramtsstudierenden."Unterrichtswissenschaft, 47 (1): 51-77.

Paetsch, Jennifer, Svenja Hammer, Annkathrin Darsow, Fränze Sophie Wagner und Timo Ehmke. 2020. »Pre-Service Teachers' Competency Development and Opportunities to Learn in Teaching Multilingual Learners in Germany. «In Teaching Content and Language in the Multilingual Classroom, herausgegeben von Svenja Hammer, Kara Mitchell Viesca und Nancy L. Commins. International Research in Policy, Perspectives, Preparation, and Practice. 124-42. New York: Routledge. 
Reusser, Kurt, Christine Pauli und Anneliese Elmer. 2011. »Berufsbezogene Überzeugungen von Lehrerinnen und Lehrern. «In Handbuch der Forschung zum Lehrerberuf, herausgegeben von Ewald Terhart, Hedda Bennewitz und Martin Rothland. 478-95. Münster: Waxmann.

Riebling, Linda. 2013. Sprachbildung im naturwissenschaftlichen Unterricht. Eine Studie im Kontext migrationsbedingter sprachlicher Heterogenität. Münster: Waxmann.

Riemer, Claudia. 2017. „DaZ und Inklusion - Gemeinsamkeiten und Unterschiede. Ein fachpolitischer Positionierungsversuch aus der Perspektive des Fachs DaF/DaZ." In Deutsch als Zweitsprache in der Lehrerbildung, herausgegeben von Michael Becker-Mrotzek, Peter Rosenberg, Christoph Schroeder und Annika Witte, 171-86. Münster: Waxmann.

Rödel, Laura, Julia Frohn und Vera Moser. 2019. »Inklusive Sprachbildung im Kontext des Forschungsprojektes FDQI-HU. «In Inklusive Sprach(en)bildung. Ein interdisziplinärer Blick auf das Verhältnis von Inklusion und Sprachbildung, herausgegeben von Laura Rödel und Toni Simon. 14-23. Bad Heilbrunn: Klinkhardt.

Rödel, Laura und Beate Lütke. 2019. »Sprachbildung im inklusiven Fachunterricht. «In Inklusives Lehren und Lernen. Allgemein- und fachdidaktische Grundlagen, herausgegeben von Julia Frohn, Ellen Brodesser, Vera Moser und Detlef Pech. 81-88. Bad Heilbrunn: Klinkhardt.,

Rost, Jürgen. 2004. »Psychometrische Modelle zur Überprüfung von Bildungsstandards anhand von Kompetenzmodellen.« Zeitschrift für Pädagogik, 50 (5): 662-78.

Sachverständigenrat deutscher Stiftungen für Integration und Migration. 2017. Vielfalt im Klassenzimmer. Wie Lehrkräfte gute Leistung fördern können.

Schwarz, Jürgen. 2016. Wilcoxon-Test: Universität Zürich, Methodenberatung. https://www.methode nberatung.uzh.ch/de/datenanalyse_spss/unterschiede/zentral/wilkoxon.html (07.11.2018).

Sigel, Irving E. 1985. »A conceptual analysis of beliefs." In Parental belief systems: The psychological consequences for children, herausgegeben von Irving E. Sigel. 345-71. New Jersey: Erlbaum.

Stiftung Mercator. 2017. Angekommen in der Migrationsgesellschaft? Grundlagen der Lehrerbildung auf dem Prüfstand. Essen.

Tracy, Rosemarie. 2014. »Mehrsprachigkeit: Vom Störfall zum Glücksfall.«In Das mehrsprachige Klassenzimmer, herausgegeben von Manfred Krifka, Joanna Blaszczal, Annette Leßmöllmann, André Meinunger, Barbara Stiebels, Rosemarie Tracy und Hubert Truckenbrodt. 13-33. Berlin und Heidelberg: Springer VS.

Tracy, Rosemarie, Dieter Thoma, Marije Michel und Daniela Ofner. 2014. „SprachKoPF: Sprachliche Kompetenzen pädagogischer Fachkräfte.« Bildungsforschung, 40: 285-87.

Walter, Oliver und Petra Stanat. 2008. »Der Zusammenhang des Migrantenanteils in Schulen mit der Lesekompetenz: Differenzierte Analysen der erweiterten Migrantenstichprobe von PISA 2003.« Zeitschrift für Erziehungswissenschaft, 11 (1): 84-105.

Weinert, Franz Emanuel. 2001. „Concept of Competence: A Conceptual Clarification.« In Defining and Selecting Key Competencies, herausgegeben von Dominique Simone Rychen und Laura Hersh Salganik. 45-65. Seattle: Hogrefe und Huber Publishers.

Weis, Mirjam, Julia Mang, Barbara Baumann und Kristina Reiss. 2018. »Zuwanderung und Erfolg aus Sicht der PISA-Studie: Ein Gesamtüberblick von 2000 bis 2015." In Handbuch Migration und Erfolg: Psychologische und sozialwissenschaftliche Aspekte, herausgegeben von Petia Genkova und Andrea Riecken. 1-14. Wiesbaden: Springer Fachmedien.

Wenzl, Thomas, Andreas Wernet und Imke Kollmer. 2017. Praxisparolen: Dekonstruktionen zum Praxiswunsch von Lehramtsstudierenden (Vol. 15). Wiesbaden: Springer VS.

Wischmeier, Inka. 2012. „Teachers' Beliefs: Überzeugungen von (Grundschul-)Lehrkräften über Schüler und Schülerinnen mit Migrationshintergrund - Theoretische Konzeption und empirische Überprüfung. «In Verstehen und Kultur, herausgegeben von Werner Wiater und Doris Manschke. 167-89. Wiesbaden: VS Verlag für Sozialwissenschaften. 


\section{Die Autorinnen}

Lisa Berkel-Otto ist wissenschaftliche Mitarbeiterin im Arbeitsbereich Sprachbildung und Mehrsprachigkeit an der Ruhr-Universität Bochum. In ihrer Dissertation erforscht sie die Hochschullehre im DSSZ-Modul. Weitere Arbeitsschwerpunkte sind u.a. Kompetenzmessung und Forschung zur Bildungssprache im Bereich der sprachlichen Bildung und Deutsch als Zweitsprache.

Kontakt:lisa.otto@rub.de

Lydia Böttger ist wissenschaftliche Mitarbeiterin im Weiterbildungsstudium »Mehrsprachigkeit, Deutsch als Zweit- und Fremdsprache« an der Universität Paderborn.

Kontakt: lydia.boettger@upb.de

Nele Fischer hat an der Leuphana Universität Lüneburg ihre Promotion zu professionellen Überzeugungen von Lehrkräften zu sprachlich-kultureller Heterogenität abgeschlossen. Aktuell arbeitet sie als Lehrkraft für die Fächer Deutsch und Englisch in Lübeck.

Kontakt: nele.fischer@leuphana.de

Tetyana Vasylyeva, Dr., ist wissenschaftliche Mitarbeiterin an der Universität Paderborn. Ihre Promotion hat sie zu Textproduktion bei bilingualen Lernern absolviert.

Kontakt: tetyana.vasylyeva@upb.de

Svenja Hammer, Dr., ist wissenschaftliche Mitarbeiterin im Arbeitsbereich Sprachbildung und Mehrsprachigkeit an der Ruhr-Universität Bochum. Ihre Promotion hat sie zu Professionellen Kompetenzen angehender Lehrkräfte im Bereich Deutsch als Zweitsprache absolviert. Weitere Arbeitsschwerpunkte sind u. a. Mehrsprachigkeit im sprachbildenden Fachunterricht.

Kontakt: svenja.hammer@googlemail.com

Constanze Niederhaus, Prof. Dr., ist Professorin für Deutsch als Zweitsprache und Mehrsprachigkeit an der Universität Paderborn. Arbeitsschwerpunkte: Deutsch als Zweitsprache und Sprachbildung im Kontext der Mehrsprachigkeit, Schul-, Bildungs- und Fachsprache, sprachbildender Fachunterricht, Unterrichten neu zugewanderter Schüler*innen.

Kontakt: constanze.niederhaus@upb.de 